\section{Die Zukunft der Pflege - 2053}

\section{Ergebnisse eines Szenarioworkshops}

von Manfred Hülsken-Giesler, PhilosophischTheologische Hochschule Vallendar, und Bernd Wiemann, deep innovation München

\begin{abstract}
Aktuelle Strategien zur Bewältigung der Herausforderung Pflege in einer Gesellschaft des langen Lebens stoßen zunehmend erkennbar an ihre Grenzen. Um tatsächlich neue Handlungsoptionen zu eröffnen, empfiehlt es sich, zunächst von bekannten und in der Regel linear verlängerten Problemlösungswegen abzulassen und - systematisch geleitet - einen erweiterten Blick in die Zukunft zu wagen. Der vorliegende Beitrag skizziert ein Projektvorhaben, mit dem mögliche Zukünfte der Pflege szenariobasiert entworfen wurden, um auf dieser Basis systematische Zukunftsdialoge zu ermöglichen und Optionen für das aktuelle Handeln abzuleiten. Fragen der Technikentwicklung und -nutzung erhalten demnach in einer transdisziplinär begründeten Pflegearbeit ganz entscheidende Bedeutung.
\end{abstract}

Current strategies to meet the challenges of care in a society of longer living are apparently reaching their limits. To actually open up new options for action, it is advisable to refrain from known approaches to solving problems and to - systematically - risk a broad view of the future. This paper outlines a project in which possible futures of care were developed on the basis of scenarios and discussed regarding their importance for systematic dialogues on the future as well as providing options for current actions. Issues of technology development and use in care are of decisive importance here.

Deutschland und immer mehr Länder der Welt sind auf dem Weg zu „Gesellschaften des langen Lebens". Die Vielfalt der Herausforderungen für den damit verbundenen gesellschaftlichen Wandel treten immer mehr in den Vordergrund. Die Bearbeitung der damit verbundenen Herausforderungen erfolgt im Bereich der langzeitorientierten Pflege derzeit vorzugsweise durch Verlängerung und Optimierung bekannter Problemlösungsstrategien, die insbesondere einer marktorientierten Perspektive entlehnt sind und betriebswirtschaftlich begründete Konzepte hervorbringen. Die Folgen sind bekannt und an dieser Stelle nur in Ausschnitten zu benennen: Privatisierung und Ausdifferenzierung der Leistungsangebote führen $\mathrm{zu}$ stetig steigendem wirtschaftlichem Wettbewerb, zunehmenden ökonomischen Restriktionen sowie steigenden Arbeitsanforderungen und -belastungen in der beruflichen Pflege. Stringente Qualitätsprüfungen auf der Basis fachlich umstrittener Qualitätskriterien erzeugen enormen Druck auf Unternehmen und Mitarbeiter. Prekäre Arbeitsverhältnisse forcieren einen ehedem demografisch bedingten Fachkräftemangel. Mangelnde Kooperationen zwischen den Versorgungssektoren und unter den Gesundheitsberufen erzeugen überdies erhebliche Schnittstellenprobleme (vgl. z. B. Brandenburg et al. 2015; Manzei/Schmiede 2014; Slotala 2011). Während also die Grenzen der bisherigen Problemlösungsansätze immer deutlicher werden, wächst auf der anderen Seite der Handlungsdruck. Vor dem Hintergrund dieser problematischen Ausgangslage haben Szenarien zur ,Zukunft von Gesundheit und Pflege" in Deutschland derzeit Konjunktur (vgl. z. B. Vollmar 2014; Rothgang et al. 2012; vbw 2012; Beckert et al. 2008). Dabei verengt sich die Perspektive der Untersuchungen jedoch häufig auf ausgesuchte Teilaspekte (Entwicklung spezieller pflegerelevanter Phänomene [z. B. Demenz], wirtschaftliche Bedeutung der Pflege, Bedeutung der technologischen Entwicklung für Gesundheit und Pflege etc.) und auf Korrekturen aktueller Fehlentwicklungen und linearer Fortschreibungen der Handlungsoptionen. Eine langfristige Orientierung, die aufzeigt, wie Strukturen der Langzeitpflege zu denken und welche Entwürfe für eine „Pflege von Morgen“ möglich wären, bleibt dagegen unscharf. Vor diesem Hintergrund skizzieren wir einen erweiterten Szenarioprozess, der das Ziel verfolgt, Perspektiven einer „Gesellschaft des langen Leben“ unter Gesichtspunkten der pflegerischen Langzeitversorgung zu entwerfen und Orientierungen und Handlungsoptionen für das heutige Handeln aufzuzeigen.

\section{Die „Reise in die Zukunft der Pflege - 2053“"}

Auf der Messe „Altenpflege 2013“ in Nürnberg wurde erstmals eine öffentliche „Zukunftsreise“ durchgeführt. ${ }^{1}$ Sie trug den Titel „Reise in die Zu- 
kunft der Pflege - 2053“, im Folgenden mit „Zukunftsreise" abgekürzt. Ausgewählte Entscheider und Verantwortliche aus verschiedenen gesellschaftlichen Teilbereichen wurden dazu eingeladen, in einem dreitägigen Workshop systematisch geleitet Vorstellungen zur Pflege im Jahr 2053 zu entwickeln und daraus Impulse für Handlungsempfehlungen für die aktuelle Situation abzuleiten. Das Ziel der „Zukunftsreise“ bestand darin, gesellschaftliche Transformationen ganzheitlich $\mathrm{zu}$ antizipieren und mit Blick auf Aspekte der Langzeitpflege in einer Gesellschaft des langen Lebens zu beschreiben und in möglichst konkreten Szenarien zu verdichten, um Orientierungen für eine gesellschaftlich ausstehende Diskussion um die Zukunft der Pflege in Deutschland zu geben. Das Vorhaben wurde als transdisziplinärer Prozess angelegt, um Impulse aus verschiedenen gesellschaftlichen Teilbereichen einzuholen, die das Handlungsfeld der Pflege beeinflussen. Insgesamt brachten elf verantwortliche Entscheider ihre Expertise in den Prozess ein.

\section{Methodisches Vorgehen}

Die „Zukunftsreise“ wurde als eine Erweiterung der Szenario-Methode angelegt, die die systematische Bearbeitung komplexer Zukunftsfragen in Aussicht stellt (vgl. z. B. Kosow et al. 2008). Da- mit wird der explorative Versuch unternommen, die Einflussfaktoren auf eine in Frage stehende Problemstellung zu identifizieren, diese in möglichen Entwicklungslinien sowie in ihrem $\mathrm{Zu}$ sammenspiel einzuschätzen, daraus Ableitungen für mögliche Zukünfte mit Blick auf die anvisierte Problemstellung zu beschreiben, um daraus Handlungsoptionen für die Gegenwart abzuleiten. Ein entscheidender Vorteil ergibt sich mit diesem Ansatz immer dann, wenn der Zeitraum der anvisierten Zukünfte so weit gewählt wird, dass sich die Ableitung von Zukunftsszenarien nicht aus der linearen Verlängerung aktuell etablierter Problemlösungsansätze im Kontext der gewählten Fragestellung ergibt, bestehende Denkmuster also aufgebrochen und durch Kreativität, vernetztes Denken und implizite Wissensbestände ersetzt werden müssen. Das konkrete Vorgehen in Ansätzen der explorativen SzenarioAnalyse wird unterschiedlich beschrieben (vgl. z. B. Popp/Schüll 2009; Beckert et al. 2008; Kosow et al. 2008; Wilms 2006; MSW NRW o. J.).

Der ,Zukunftsreise“ wird ein modifizierter Ansatz mit acht Schritten zugrunde gelegt: Problem- und Umfeldanalyse, Gegenwartsanalyse, Einflussanalyse, Projektionsbildung, Alternativenbündelung, Szenariointerpretation, Störfallanalyse, Konsequenzenanalyse verbunden mit Maßnahmenplanung (Abb. 1).

Abb. 1: Methodische Vorgehen in der „Zukunftsreise“ (modifizierter Szenarioprozess)

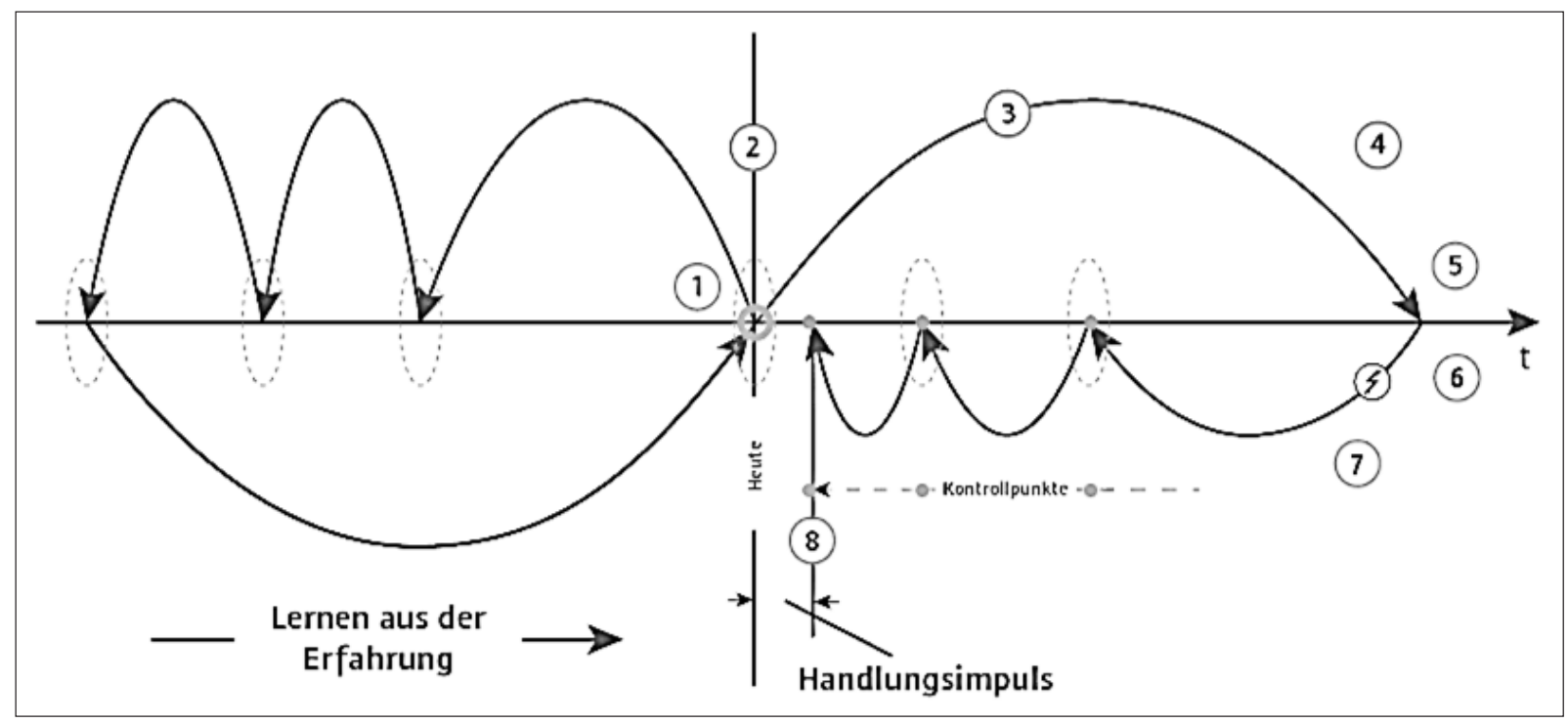

Quelle: Eigene Darstellung 
Phase 1, Problem- und Umfeldanalyse, wurde bereits im Vorfeld der Expertenworkshops erarbeitet und den Workshopteilnehmern in Form eines Impulspapiers zugeleitet, das einen Rückblick auf die Vergangenheit der Langzeitpflege in Deutschland erlaubt und die aktuellen Problemlagen systematisch ableitet. Phase 2 setzt sich mit einer Gegenwartsbeschreibung, also dem Status der Pflege im Jahr 2013 auseinander. In Phase 3, Einflussanalyse, geht es darum, aktuelle Einflussbereiche und Einflussfaktoren auf die Pflege zu identifizieren sowie ihr Systemverhalten und relevante Vernetzungen zu erkennen. Daraus lassen sich treibende und getriebene Kräfte in Bezug auf den Untersuchungsgegenstand identifizieren und bewerten (Sensitivitätsanalyse). Phase 4, Projektionsbildung, fokussiert darauf, Zukunftsentwicklungen für die identifizierten Einflussfaktoren zu generieren. Die Benennung von Alternativen zukünftiger Ausprägungen für jede Projektion, die nachvollziehbar zu begründen sind, ist die Grundlage für das Zusammensetzen der Szenarien im Folgeschritt. Das Ziel der folgenden Phase 5, Alternativenbündelung, besteht darin, die erarbeiteten Alternativen in konsistente und nachvollziehbare SzenarioStrukturen zusammenzufügen, die in der durchgeführten Studie auf zwei Extrem-Szenarien begrenzt wurden: Szenario A: ,Sozial nachhaltiges Netzwerk - Jeder MUSS ran!“ und Szenario B: „Leben ohne Alter“ (Abb. 3 und Abb. 4). Aus den Extrem-Szenarien wurde zusätzlich ein TrendSzenario gebildet, das Schnittmengen der vorgenannten Szenarien enthält und damit eine höhere Aussagen-Robustheit vermittelt. In Phase 6, Szenario-Interpretation, werden die skizzierten Szenarien lebensnah und möglichst plausibel in ihrer Dynamik und in ihren Entwicklungsprozessen beschrieben. Damit soll eine Vorstellung vom täglichen Leben im Jahre 2053 unter besonderer Berücksichtigung von Fragen der Pflege ermöglicht werden, damit die Szenarien nachvollziehbar und für die weitere Bearbeitung handhabbar sind. Das Ziel besteht in dieser Phase nicht darin, die Szenarien vergleichend zu bewerten. Mit der Störfallanalyse in Phase 7 werden mögliche Störereignisse identifiziert und in ihren Auswirkungen analysiert, die diese Zukunfts-Szenarien verhindern könnten. Damit soll der Umgang mit
Unsicherheiten als Planungskalkül integriert und Sensibilität für „frühe Signale“ geschärft werde. Dazu gehören z. B. globale Entwicklungen, Epidemien, wirtschaftliche Umbrüche aber auch $\mathrm{Pa}$ radigmenwechsel im Bereich von Technik oder Ethik. In Phase 8, der Konsequenzenanalyse mit Maßnahmenplanung werden Chancen und Risiken analysiert und beschrieben, die mit den erarbeiteten Szenarien verbunden sind. Besonders werden konkrete Ansätze gesucht, diese Zukünfte zu gestalten, identifizierte Chancen optimal zu nutzen und erkannte Risiken nicht nur zu minimieren, sondern bestenfalls in neue Chancen umzuwandeln. Schließlich besteht das Ziel der letzten Phase 8 auch in der Maßnahmenplanung, um Leitlinien zu konzipieren, die unter den identifizierten Szenario-Rahmenbedingungen greifen. Im Kontext der durchgeführten Studie konnte für die letzten zwei Phasen (Störfallanalyse, Konsequenzenanalyse mit Maßnahmenplanung) lediglich vorläufiges Material gesammelt werden. Die weitere Ausarbeitung erfolgt in entsprechenden Folgeprojekten.

\section{Ergebnisse und Analysen auf dem Weg zur Szenariobildung (Phase 1-4)}

Die Vielfalt der Ergebnisse der „Reise in die Zukunft der Pflege - 2053“ kann an dieser Stelle nur zusammenfassend präsentiert werden, eine differenziertere Vorstellung entlang der benannten Arbeitsschritte befindet sich in Vorbereitung (vgl. deep innovation GmbH 2015a; deep innovation $\mathrm{GmbH} 2015 b)$.

\subsection{Ergebnisse Phase 1: Die Evolution der Pflege}

In der ersten Phase geht es um eine systematische Erfassung der Historie der Langzeitpflege. Dazu wurden zwei Systematisierungsverfahren entwickelt, die in alle weiteren Phasen eingeflossen sind:

- Bestimmung und Systematisierung der evolutionären Entwicklung der Pflege,

- Charakterisierung von Determinanten der Pflege und ihre Entwicklung. 


\section{Evolutionäre Entwicklung der Pflege}

Auf der Basis eines evolutionären Verständnisses der Pflegehistorie wurde die Entwicklung über ca. 2000 Jahre grob in drei zeitliche Abschnitte untergliedert:

- Pflege im vormodernen Pflegesystem (Antike bis 19. Jahrhundert),

- Pflege im sozialstaatlichen Pflegesystem (19. Jahrhundert bis 1995),

- Pflege im vermarktlichten Pflegesystem (1995 bis heute).

Diese Einteilung begründet sich entlang grundlegender Umwälzungen im Kultur- und Berufsfeld, die sich stichwortartig unter den Aspekten: christlich-metaphysische Begründung und beginnende Institutionalisierung der Pflege (vormodernes Pflegesystem), sozialpolitisch motivierte und beruflich institutionalisierte Pflege (sozialstaatliches Pflegesystem) und marktwirtschaftlich motivierte und professionell institutionalisierte Pflege (vermarktlichtes Pflegesystem) zusammenführen lassen. Auf dieser Basis wurde eine zusammenhängende Beschreibung der Evolution der Pflege bis heute erstellt, aus deren Merkmalen Prinzipien erkennbar und Schlüsse für die Weiterentwicklung gezogen werden können.

\section{Die Determinanten der Pflege}

Ergänzend wurde ein Vorschlag erarbeitet, den Untersuchungsgegenstand Pflege aus der Sicht von sechs verschiedenen Perspektiven (Determinanten) zu analysieren. Dadurch können die Grundprinzipen und ihre gesellschaftlichen Konsequenzen als relevante Veränderungen über alle Abschnitte hinweg herausgearbeitet werden: Mit der Determinante Leitbilder der Pflege sind demnach die zentralen Handlungsrationalitäten der Pflegearbeit in einer jeweiligen historischen Phase in den Blick zu nehmen. Die Entwicklung von Wertesystemen der Pflege verdeutlicht wandelnde Motive des pflegerischen Handelns. Pflege als gesellschaftliches Handeln stellt sich in der Determinante Öffentliche Wahrnehmung der Pflege in den jeweiligen Entwicklungsphasen sehr unterschiedlich dar und erfährt verschiedene Formen der Anerkennung. Unter Organisation der Pflege lässt sich die Entwicklung der gesellschaftlichen und institutionellen Rahmenbedingungen der Pflege verfolgen, um schließlich mit Gegenstand der Pflege und Wissen der Pflege historische Transformationen im Berufsfeld selber aufzuzeigen.

\subsection{Ergebnisse Phase 2: Die Standortbestimmung der Pflege 2013}

Mit Blick auf die aktuelle Situation (Phase 2) identifizierten die Expertinnen und Experten drei wesentliche Einflussfelder, die die Entwicklung der Pflege in Deutschland prägen: a) Solidargemeinschaft und marktwirtschaftliche Entwicklung, b) wissenschaftliche und technologische Entwicklung/Infrastruktur, c) gesellschaftliche und wirtschaftliche Entwicklung.

Unter Gesichtspunkten von Solidargemeinschaft und marktwirtschaftlicher Entwicklung wird darauf verwiesen, dass sich Initiativen zur Stärkung der Solidargemeinschaft aktuell zwar dynamisch in Diskussion und, regional begrenzt, auch in modellhafter Erprobung befinden, diese jedoch nach wie vor durch eine Organisation von Pflege im Spannungsfeld von sozialgesetzgeberischen und marktbedingten Rahmungen überlagert werden. Private wie gemeinnützige Anbieter fokussieren vorzugsweise auf isolierte Bedarfe der Nachfrager, lokal vernetzte Angebote sind seltener zu finden. Die Nachfrage erfolgt dabei heute zunehmend von informierten und kritischen Leistungsnehmern, die an individuellen und flexiblen Dienstleistungen interessiert sind und über ein hohes Kosten- und Leistungsbewusstsein verfügen.

Die wissenschaftlich-technologische Entwicklung wirkt sich derzeit wesentlich über Veränderungen und Innovationen im Bereich der medizinisch-gesundheitlichen Versorgung, der Technik- und Technologieentwicklung sowie der Wohninfrastruktur auf die Pflege aus. Medizinische Spezialisierungen (z. B. im Bereich der Onkologie, Kardiologie, Diabetologie, Palliativmedizin) sind von Relevanz, insofern sie die Unterscheidung zwischen Allgemeinmedizin und Altersmedizin zunehmend einebnen. Unklar ist jedoch, inwieweit sich diese Entwicklung auf aktuelle Professionalisierungsprozesse der Gesundheitsberufe im Allgemeinen und der Pflege im Besonderen auswirken. Die technologische Entwicklung (z. B. Assistive 
Technologien, Robotik, I\&K-Technologien) birgt durch Möglichkeiten der unmittelbaren Unterstützung des Leistungsgeschehens, der Weiterentwicklung von Arbeitsprozessen sowie der Vernetzung relevanter Akteursgruppen Potenziale, ,Raum und Zeit" für die Pflege zu schaffen (technologische Personalisierung), zeigt aktuell aber auch Tendenzen einer technologischen Fragmentierung, insofern Technologien zum Einsatz kommen, die lediglich Teilaspekte der Pflegearbeit unterstützen. Überdies sind Gefahren einer technologischen Deprofessionalisierung erkennbar, insofern sich Effekte der Entfremdung zwischen Pflegenden und Hilfeempfängern durch reduzierte Kontaktzeiten einerseits und der Überformungen von Entscheidungsprozessen durch akteursübergreifende Datenflüsse andererseits andeuten. Auf der Ebene der Infrastruktur der pflegerischen Versorgung ist $\mathrm{zu}$ beobachten, dass Pflegearbeit zunehmend in neuen Umgebungen und Konstellationen gedacht wird. Innovative Konzepte etwa des generationenübergreifenden, barrierefreien und/oder intelligenten Wohnens suchen das Potenzial neuer Sozialräume zur Unterstützung der Pflegearbeit auszuloten. Kritisch wird diskutiert, dass sich entsprechende Strukturen derzeit vorzugsweise ,drittmittelgetrieben" realisieren, die Analyse und Bewertung von Ausgangssituationen und Innovationsansätzen jedoch unter Gesichtspunkten der Einwerbung von Fördermitteln ggf. einer eigenen Logik folgen und damit die Gefahr besteht, dass die konkreten Erfordernisse und Unterstützungsbedarfe im Versorgungsfeld aus dem Blick geraten.

Die gesellschaftliche und wirtschaftliche Entwicklung ist heute nicht mehr unabhängig von tief greifenden Umwälzungen einer modernisierten und globalisierten westlichen $\mathrm{Zi}$ vilisation zu diskutieren. Demografische und epidemiologische Veränderungen, Individualisierungsprozesse, Wertewandel, Rationalisierungs- und Globalisierungstendenzen u. a. m. greifen ineinander, bedingen und potenzieren sich. Mit diesen Prozessen sind Chancen und Gefahren verbunden. Mit Blick auf Fragen der Pflege kommen dabei Transformationen von der Industrie- zur Wissensgesellschaft ebenso zum Tragen wie die Auflösung ehedem gesamtgesellschaftlich getragener Wertvorstellungen in Richtung eines Wertepluralismus. Digitalisie- rung und Computerisierung führen zu immensen Wissens- und Informationszuwächsen, die heute - auch in der Pflege - Probleme der Informationsselektion und -bewertung erzeugen und vor diesem Hintergrund spezifische verarbeitungsfreundliche und systemkompatible Wissensformen und -bestände begünstigen. Auch mit Blick auf die Werteentwicklung lassen sich ambivalente Prozesse beobachten, die etwa am Beispiel von Individualisierung und Flexibilisierung von Lebensstilen einerseits und zunehmendem Bewusstsein für gesellschaftliche und soziale Verantwortung andererseits oder auch von paradoxen Bewegungen zwischen Globalisierung und Lokalisierung der Lebensführung zu skizzieren sind. Auf der politischen Ebene erfolgt eine inhaltliche Neubestimmung von (sozialrechtlich legitimer) Pflege bislang eher zögerlich, die Bemühungen konzentrieren sich einerseits darauf, Anreize für mehr Eigenverantwortung auf Seiten der Bürger zu schaffen, und andererseits Qualitätsentwicklung im Pflegewesen voranzutreiben. Unter wirtschaftlichen Gesichtspunkten ist die Entwicklung zu einer „Gesellschaft des langen Lebens" vor allem mit der Erschließung neuer Märkte verbunden. Der Pflegemarkt wird als Wachstumsmarkt betrachtet, dessen Besonderheit allerdings darin besteht, dass Teile der Dienstleistung über das Dreieck „Leistungsanbieter“, „Leistungsnehmer“ und „Sozialversicherungsträger" auszuhandeln sind, die Prinzipien der freien Marktwirtschaft hier also an Grenzen stoßen. Neben inhaltlich motivierten Aspekten einer verbesserten interdisziplinären Zusammenarbeit lassen sich in diesem Umstand auch neue Verflechtungen zwischen Anbietern professioneller Pflegeleistungen einerseits und medizinischen, medizintechnischen, pharmazeutischen sowie weiteren Anbietern andererseits beobachten. Um den neu etablierten Pflegemarkt ist vor diesem Hintergrund eine Lobby entstanden, die ihre Interessen in die zukünftige Entwicklung der Pflege einzubringen versucht.

Um die aktuellen Problemstellungen 2013 $\mathrm{zu}$ charakterisieren und $\mathrm{zu}$ pointieren, wurden mittels 20 Kontradiktionen die heute erkennbaren Widersprüche zwischen Anspruch der Pflege und Pflegerealität gegenübergestellt und analysiert. Die Ergebnisse dieser Arbeiten fließen in 
Abb. 2: Sensitivitätsdiagramm Pflege

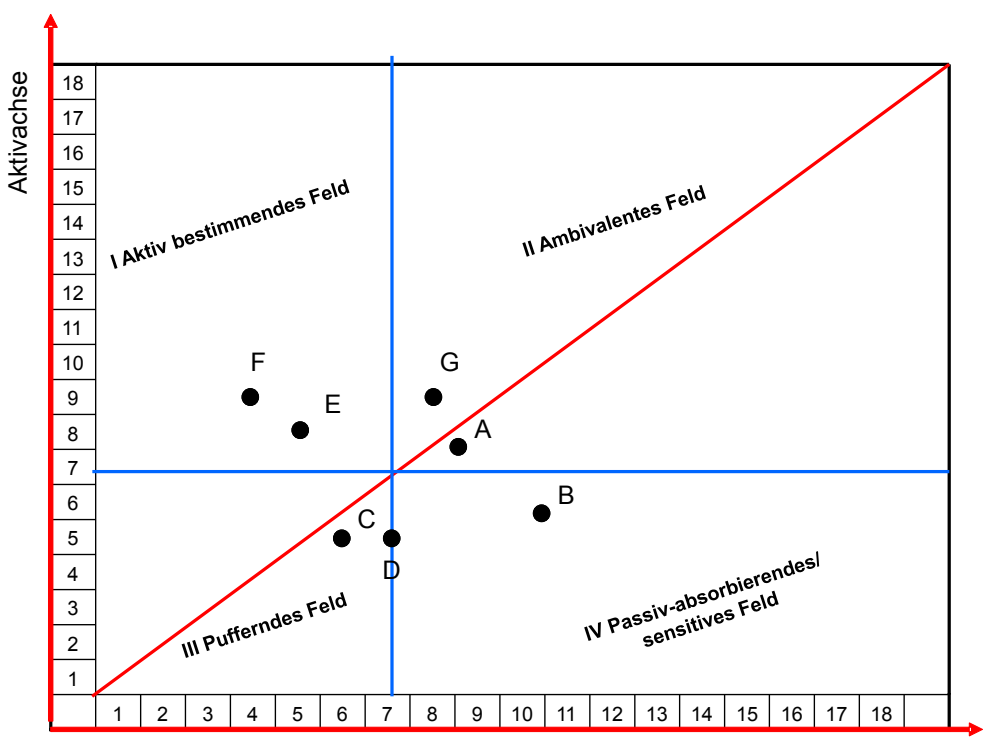

Passivachse

$\mathrm{A}=$ Anbieter; $\mathrm{B}=$ Nachfrager; $\mathrm{C}=$ Technologie/Infrastruktur;

$\mathrm{D}=$ Medizin; $\mathrm{E}=$ Wirtschaft; $\mathrm{F}=$ Gesellschaft; $\mathrm{G}=$ Politik/Gesetzgebung

Quelle: Eigene Darstellung

die Gestaltung der Zukunftsprojektionen ein, sollen aber auch der Fortschrittsbeschreibung in der Pflege dienen. Diese Standortbestimmung der Pflege 2013 stellt den Ausgangspunkt der Entwicklung hin zu den Szenarien für 2053 dar.

\subsection{Ergebnisse Phase 3: Einflussanalyse, Sensitivitätsanalyse (treibende und folgende Faktoren)}

Die in der Standortbestimmung skizzierten Einflussfaktoren auf die Pflege in Deutschland wurden im Rahmen der „Sensitivitätsanalyse“ (Phase 3) in Beziehung gesetzt und von den Expertinnen und Experten (quantitativ) bewertet. Es wurden die treibenden, die abhängigen und die blockierenden Faktoren ermittelt. Die Überführung dieser Einschätzungen in eine Sensitivitätsmatrix erlaubt Feststellungen darüber, welches die gestaltenden Kräfte sind und wie sensibel das Feld der Pflege auf Veränderungen wirkt. Dabei wurde deutlich, dass den gesellschaftlichen, politischen und wirtschaftlichen Faktoren eine besondere Bedeutung in der aktiven Beeinflussung der Pflege zukommt. Das Verhalten von Anbie- tern und Nachfragern ist dagegen besonders abhängig gegenüber diesen aktiven Einflussfaktoren, sie bleiben hinsichtlich der Gestaltung des Handlungsfeldes eher passiv (Abb. 2).

\subsection{Ergebnisse Phase 4: Deskriptoren und Projektionen}

Die Phase 4 der ,Zukunftsreise" widmet sich auf der Basis der identifizierten Einfluss-, Abhängigkeits- und Problemfelder von 2013 der Entwicklung von Projektionen für das Jahr 2053. Dazu wurden die Eingangsgrößen von 2013 mit Blick auf ihre Veränderungsdynamik bis 2053 untersucht und zweistufig in alternativen Paarungen detaillierter Projektionen beschrieben. Tabelle 1 gibt die 26 alternativen Projektionen wieder, aus denen in der folgenden Phase 5 durch Auswahl die konsistenten Szenarien für 2053 gebündelt wurden:

Tab. 1: Projektionsbildung und Alternativenbündelung

\begin{tabular}{|c|c|c|c|}
\hline Einflussfeld & Alternative 1 & $v s$ & Alternative 2 \\
\hline \multirow[t]{2}{*}{ Gesellschaft } & $\begin{array}{l}\text { Professionelle } \\
\text { Pflege }\end{array}$ & vs. & $\begin{array}{l}\text { zivilgesellschaftli- } \\
\text { che Pflege }\end{array}$ \\
\hline & Multi Value System & vs. & $\begin{array}{l}\text { Einheitliches Werte- } \\
\text { system }\end{array}$ \\
\hline \multirow[t]{2}{*}{ Wirtschaft } & $\begin{array}{l}\text { Solidarökon. Sys- } \\
\text { tem }\end{array}$ & vs. & $\begin{array}{l}\text { Freie Marktwirt- } \\
\text { schaft }\end{array}$ \\
\hline & Kollektives Modell & vs. & $\begin{array}{l}\text { Individuelles Mo- } \\
\text { dell }\end{array}$ \\
\hline \multirow[t]{2}{*}{ Politik } & Wohlfahrtsmodell & vs. & Marktmodell \\
\hline & Zentralmodell & vs. & Lokalmodell \\
\hline Medizin & Mit dem Alter leben & vs. & $\begin{array}{l}\text { Abschaffung des } \\
\text { Alters }\end{array}$ \\
\hline \multirow[t]{2}{*}{ Technologie } & Inklusion & vs. & Exklusion \\
\hline & Technik als Sklave & vs. & Technik als Master \\
\hline \multirow[t]{2}{*}{ Anbieter } & Globale Träger & vs. & Lokale Träger \\
\hline & $\begin{array}{l}\text { Komplette } \\
\text { Verheimung }\end{array}$ & vs. & $\begin{array}{l}\text { Komplette Ambu- } \\
\text { lantisierung }\end{array}$ \\
\hline \multirow[t]{2}{*}{ Nachfrager } & $\begin{array}{l}\text { Autonomie \& } \\
\text { Teilhabe }\end{array}$ & vs. & Satt und Sauber \\
\hline & Nachfrage gering & vs. & Nachfrage hoch \\
\hline
\end{tabular}

Quelle: Eigene Darstellung 
Abb. 3: Szenario „Jeder MUSS ran“

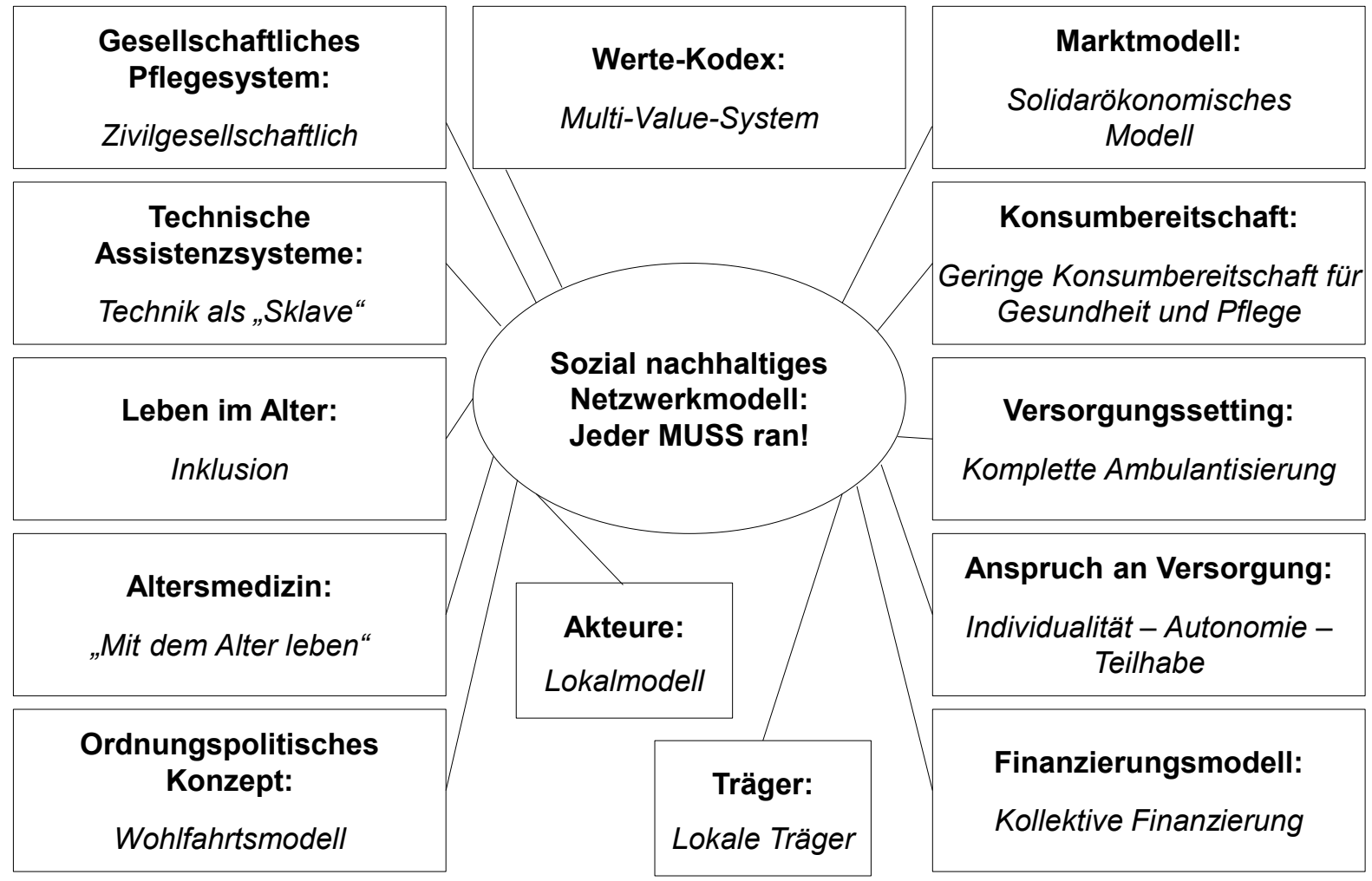

Quelle: Eigene Darstellung

\section{Ergebnisse Phase 5: Die Szenarien}

Zur Ausarbeitung der Szenarien im Rahmen der „Szenariobildung“ werden die zentralen Deskriptoren der Projektionen in Beziehung gesetzt und gebündelt. Vor diesem Hintergrund formulierte die Expertengruppe zwei konträre, aber in sich konsistente und intersubjektiv nachvollziehbare Szenarien, die die mögliche Entwicklung der Pflege in 2053 skizzieren: Szenario A: „Sozial nachhaltiges Netzwerk - Jeder MUSS ran!" und Szenario B: „Leben ohne Alter“ (Abb. 3 und Abb. 4)

\subsection{Szenario A: „Sozial nachhaltiges Netzwerk - Jeder MUSS ran!“}

Das Szenario A „Sozial nachhaltiges Netzwerk Jeder MUSS ran!“ beschreibt eine Gesellschaft, in der es gelungen ist, ein Bewusstsein für die Bedeutung von sozialem Engagement und sozialer Verantwortung zu etablieren und die Gesellschaftsmitglieder dazu zu motivieren, dieses aktiv und freiwillig einzubringen. Herausfor- derungen der Pflege werden im Rahmen eines zivilgesellschaftlich orientierten Pflegesystems bewältigt, bürgerschaftliches Engagement erhält Vorrang vor professioneller Initiative, und diese greift erst, wenn netzwerkgestützte informelle Arbeit an ihre Grenzen kommt.

Im Mittelpunkt steht der Versuch, gesellschaftliche Teilhabe durch die aktive Einbindung aller Bürger in die Etablierung einer modernen Sozialgesellschaft zu erreichen. Inklusion, sowohl der Alten, Gebrechlichen und Pflegebedürftigen in das soziale Leben, als auch der Jungen und Leistungsfähigen in die konkrete Bewältigung sozialer Problemstellungen, gilt in dieser Gesellschaft als Recht und als Pflicht für jeden Bürger. Dabei wird akzeptiert, dass sich die Menschen sehr unterschiedlichen Wertesystemen verpflichtet fühlen und die Präferenzen für die konkrete Ausgestaltung einer selbstbestimmten Lebensführung (auch bei Hilfebedarf) damit sehr verschieden ausfallen. Dies gilt auch für die potenziellen Helfer, die ihr soziales Handlungsfeld (Pflege, Erziehung, soziale Arbeit etc.) und auch 
das konkrete Gegenüber einer Hilfeleistung frei wählen können und sollen. Soziales Engagement kann freiwillig erbracht, sollten entsprechende Initiativen bei Bürgern aber nicht erkennbar sein, auch durch Repressionen durchgesetzt werden. Die Wiederbelebung eines sozialen Pflichtjahres und/oder die gesellschaftliche Ächtung von Bürgern, die soziales Engagement grundsätzlich verweigern, wären denkbare Varianten zur gesellschaftlichen Durchsetzung dieser Vorstellung. Es wird ein verbindlicher Mindestzeitraum definiert, für den sich jeder Bürger sozialen Aktivitäten zu widmen hat. Pflegearbeit wird vorrangig durch zivilgesellschaftlich organisierte Gruppen geleistet und kann damit kostengünstig und individuell angeboten werden. Pflegearbeit wird grundsätzlich gemeindenah, in der häuslichen Umgebung pflegebedürftiger Menschen erbracht. Familienmodelle, ehrenamtliche Aktivitäten, nachbarschaftliche Solidarität, Mehrgenerationeninitiativen, lokale Interessen- und Nutzergemeinschaften - Ansätze dieser Art differenzieren sich dazu je nach lokalen Gegebenheiten zu unterschiedlichsten Unterstützungssystemen aus.

Neue Technologien können aufgrund hoher Nutzerakzeptanz und -kompetenz sowie staatlicher Förderung gut in die Netzwerkarbeit integriert werden und unterstützen sowohl Aspekte der unmittelbaren Versorgung als auch der Qualitätssicherung.

Um gesellschaftliches Engagement im Rahmen dieses Modells sinnvoll zu bündeln, erhält die Entwicklung und Aufrechterhaltung von lokalen Netzwerken eine große Bedeutung. Die Organisation, Koordination und Beratung lokaler sozialer Netzwerke wird dabei als zentrale Aufgabe der Kommunen betrachtet, der sie mit Unterstützung gemeinnütziger Leistungsanbieter nachkommen. Die Kommunen zeichnen dafür verantwortlich, dass sichergestellt ist, dass alle Bürger, unabhängig vom gesundheitlichen oder sozialen Status, ihrer Verpflichtung zum bürgerschaftlichen Engagement nachkommen. Sie unterstützen darüber hinaus die soziale Netzwerkarbeit durch Bereitstellung der erforderlichen (z. B. technischen) Infrastruktur und sichern die Finanzierung von notwendigen Materialien (z. B. Pflegehilfsmittel). Die Bereitstellung dieser Leistungen über die Kommunen sorgt dafür, dass die jeweiligen
Modelle die lokalen Besonderheiten und gewachsenen Traditionen berücksichtigen und in enger Abstimmung mit den Bürgern erstellt werden.

Moderner Technologie kommt im Kontext netzwerkgestützter Hilfeleistungen eine besondere Funktion zu: Sie trägt insbesondere dazu bei, dass die komplexe Abstimmung von Unterstützungsbedarfen und Unterstützungsangeboten optimiert werden kann. Eine Gesellschaft, in der „sozial nachhaltige Netzwerke“ eine tragende Funktion übernehmen, hat gelernt, moderne Technologien in einer Weise zu entwickeln und zu verwenden, die primär der Unterstützung der zentralen Aufgaben des gesellschaftlichen Handelns dient. Entwicklung und Verwendung moderner Technologien erfolgen grundsätzlich entlang gesellschaftlich formulierter Ziele. Technologien gelten erst dann als innovativ, wenn sie dazu führen, dass die Gesellschaft der Realisierung ihrer Zentralziele näher kommt.

In der ,Jeder MUSS ran“-Gesellschaft geht es darum, dass jede Generation und ganz konkret jeder Bürger soziales Engagement in das gesellschaftliche Leben einbringt, andererseits aber auch von der sozial engagierten Gesellschaft profitieren kann. Jüngere Generationen profitieren von der Lebenserfahrung der Älteren, die gesellschaftlich an Bedeutung gewinnt. Darüber hinaus bringen sich Ältere aber auch ganz konkret in das gesellschaftliche Leben ein und unterstützen mit ihren vielfältigen Erfahrungen das Alltagsleben jüngerer Familien. Die älteren Generationen profitieren, indem ihnen die Möglichkeit gegeben wird, ein Leben und Sterben in der biografisch gewachsenen bzw. individuell gewünschten Umgebung bei hoher gesellschaftlicher Integration zu führen und dabei ggf. sinnstiftende Aktivitäten bis ins hohe Alter ausüben zu können. Selbst pflegebedürftige Menschen können vor diesem Hintergrund noch für ein soziales gesellschaftliches Engagement gewonnen werden. Im Mittelpunkt steht der Gedanke, Alter und schließlich auch Tod und Sterben als natürliche Phänomene zu akzeptieren und in das gesellschaftliche Leben zu integrieren. Vor diesem Hintergrund wird den Menschen ermöglicht, den Altersprozess, ggf. mit Unterstützung von Rehabilitations- und Versorgungsteams, bewusst zu gestalten,, ggf. auch zu genießen und sich aktiv mit dem eigenen Sterbeprozess auseinanderzusetzen. 
Die Bereitstellung freiwilliger sozialer Ressourcen führt dazu, dass die Engführung der Pflegedebatte auf rein finanzielle Aspekte überwunden wird. Die Einsicht, dass Pflegearbeit nicht auf somatisch-funktionale Aspekte zu reduzieren ist, sondern komplexe soziale und lebensweltlichbiografische Facetten wesentlich einzubinden sind, findet gesellschaftliche Anerkennung. Es wird jedoch davon ausgegangen, dass entsprechende Aktivitäten (Prävention, Gesundheitsförderung, Unterstützungsleistungen etc.) über kollektive Sach- und Geldleistungen und nicht über den privaten Konsum zu finanzieren sind. Das solidarökonomische Marktmodell der Pflege wird wesentlich über kollektive Finanzierungsleistungen sichergestellt. Jeder Bürger zahlt in eine Versicherung entsprechend seiner wirtschaftlichen Leistungsfähigkeit ein und erhält umgekehrt auch Leistungen aus dem Solidarsystem. Die Versorgung eines jeden wird damit durch aktives soziales und finanzielles Engagement eines jeden Bürger der Solidargemeinschaft sichergestellt - Pflegeleistungen sind zwar innerhalb des jeweiligen
Unterstützungsnetzwerkes individuell auszuhandeln, es wird aber darauf Wert gelegt, dass dabei individuelle Präferenzen nicht zu qualitativen Unterschieden in der Versorgung führen.

\subsection{Das Szenario B: „Leben ohne Alter“}

Das Szenario „Leben ohne Alter“ kann aufgrund folgender Konstellation der projizierten zentralen Einflussfaktoren beschrieben werden (Abb. 4).

Es entwickelt sich eine Gesellschaft, in der Leistungsbereitschaft und Leistungsfähigkeit höchste Priorität erhalten und alle Beteiligten zu besonderen Investitionen in Gesundheit und Vitalität bereit sind. Leistungsfähigkeit und Vitalität gehören zum Habitus des modernen Menschen, der sich selbst auf Jugend, Gesundheit, Dynamik und Leistungsbereitschaft verpflichtet. Das gesellschaftliche Leben sowie gesellschaftliche Investitionen konzentrieren sich nahezu ausschließlich auf die Lebensphasen und Lebensbereiche, an denen aktive, leistungsstarke und vitale Bürger beteiligt sind.

Abb. 4: Einflussgrößen Szenario „Leben ohne Alter66

\begin{tabular}{|c|}
\hline $\begin{array}{c}\text { Gesellschaftliches } \\
\text { Pflegesystem: } \\
\text { Professionelle Pflege }\end{array}$ \\
\hline $\begin{array}{c}\text { Technische } \\
\text { Assistenzsysteme: } \\
\text { Technik als ,Master" }\end{array}$ \\
\hline Leben im Alter: \\
Exklusion \\
\hline Altersmedizin: \\
"Abschaffung des Alters“ \\
\hline Ordnungspolitisches \\
Konzept: \\
Marktmodell
\end{tabular}

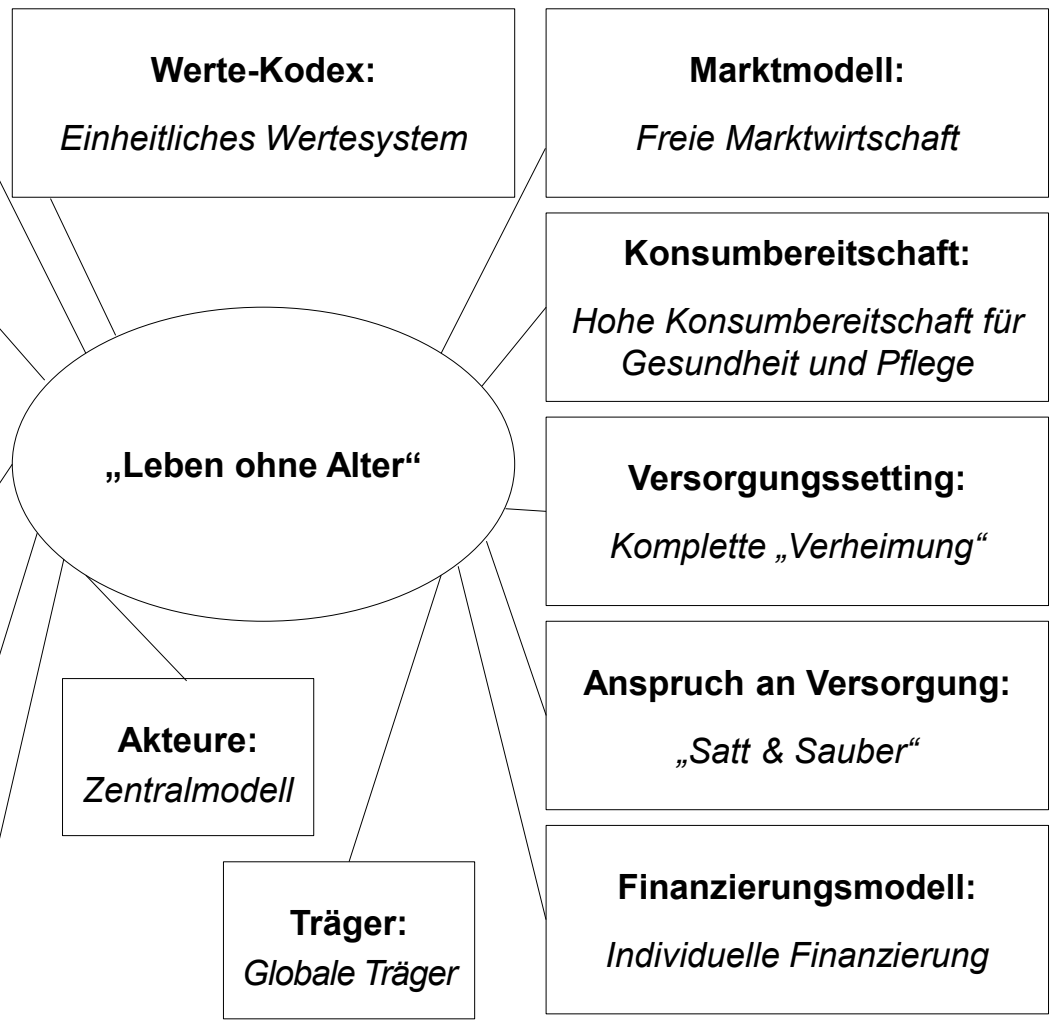

Quelle: Eigene Darstellung 
Öffentlich sichtbare Alterserscheinungen sind in dieser Gesellschaft überwunden, gesellschaftlich gilt das Alter als abgeschafft, es ist in der Öffentlichkeit nicht mehr präsent und gesellschaftlich auch nicht erwünscht. Eine hoch potente lebenswissenschaftlich orientierte Forschung unterstützt diese Entwicklung durch Bereitstellung von technologischen und medizinischen Innovationen im Bereich der funktionalen und ästhetischen Optimierung des menschlichen Körpers (z. B. „Functional Food“, Transplantationen, technische Implantate, Genmanipulationen etc.). Die Lebenserwartung kann mit diesem Modell deutlich verlängert werden. Krankheit, Alter und Pflege werden als gesellschaftliche Bürde erfahren, gesellschaftliche Aufwendungen für diese Phänomene werden entsprechend auf ein Minimum reduziert.

Innovative Technologien spielen in diesem Gesellschaftssystem eine zentrale Rolle, insofern sie u. a. auch für den reibungslosen Datenfluss zur Kontrolle und Steuerung von Vital- und Gesundheitsdaten erforderlich sind. Technologische Systeme sind weiterhin zentral an Entscheidungsprozessen zur Bestimmung des ,gesellschaftlichen Wertes" eines konkreten Menschen beteiligt und geben automatisiert Hinweise zur „Gesellschaftstauglichkeit" der Individuen.

Der einzelne Bürger ist grundsätzlich aufgefordert, seine Leistungsfähigkeit permanent zu erhalten und ggf. auch nachzuweisen. Medizinische Rehabilitationsprogramme sind zwar freiwillig, aber gesellschaftlich hoch akzeptiert, sie werden von den Bürgern rege in Anspruch genommen, um sich bei gesundheitlichen Beeinträchtigungen rasch wieder in das Berufsleben zu integrieren. Präventions- und Gesundheitsleistungen werden über einen freien und globalen Gesundheitsmarkt angeboten, der vielfältige kommerzielle Angebote zur Unterstützung und zum Erhalt der Leistungsfähigkeit hervorbringt und damit die Sozialkassen erheblich entlastet. Freier Wettbewerb und private Finanzierung von Vital- und Gesundheitsleistungen führen auch zur Ausdifferenzierung und Weiterentwicklung von neuen Gesundheitsberufen (z. B. Revitalisierungscoach etc.). Die Konsumbereitschaft für Vital- und Gesundheitsleistungen auf Seiten der Bürger ist hoch und stabilisiert den neu entstehenden Markt.
Im Aufbau ist ein hoch differenziertes, medizinisch optimiertes Revitalisierungssystem auf der Basis eines technikgestützten Revitalisierungsmonitorings. Ein implantierter Revitalisierungsmonitor erkennt im Einzelfall, wann ein Mensch einen Revitalisierungsschub benötigt und leitet die Informationen an ein Care-Center weiter. Damit wird es möglich, die periodisch notwendigen Revitalisierungsbedarfe zu erkennen und überdies einzelfallorientierte Maßnahmen anzubieten, die das Leben bei spontanen Revitalisierungsbedarfen substanziell verbessern und die erwünschte Leistungsfähigkeit herstellen. Revitalisierungsmaßnahmen sind aus medizinischen Gründen regelmäßig durchzuführen, ein Verzicht darauf führt zu einer schnellen Degradation der Lebensfunktionen.

Reagieren die Vitalparameter einer Person bei einem Revitalisierungsprozess nicht wie erwünscht, wird mit dem Einverständnis der betreffenden Person der Sterbeprozesses eingeleitet, der zeitlich auf eine kurze Periode reduziert werden kann. Diese kurze Sterbephase wird so angenehm wie möglich (,Restlebensglück“), aber auch gesellschaftlich so unauffällig wie möglich gestaltet.

Menschen, die sich grundsätzlich nicht in das gesellschaftlich etablierte Revitalisierungssystem einbinden wollen, haben die Möglichkeit, auf eine „Lebensinsel“" verbracht zu werden. Dies ist ein Lebensraum, der international sanktioniert und genutzt wird, wirtschaftlich unabhängig ist und in dem keine Revitalisierungen erfolgen. Personen, die diesen Weg gehen, sind auf diese Weise gesellschaftlich isoliert, exkludiert und öffentlich unsichtbar gemacht.

In der Übergangsphase bis zur Etablierung des Revitalisierungssystems bis 2053 ist alten, gebrechlichen und pflegebedürftigen Menschen - aufgrund mangelnder Leistungsfähigkeit - das Recht auf Wertschätzung abhandengekommen. Eine „Satt \& Sauber-Pflege“ bis zum Lebensende entspricht dem gesellschaftlichen Status dieser Menschen. Gleichzeitig wird aber für eine gute Versorgung und ein angemessenes soziales Umfeld von Sterbenden gesorgt. Sterben und Tod entziehen sich der öffentlichen Wahrnehmung und sind gleichzeitig auch nicht mehr Teil des gesellschaftlichen Lebens. Globale Träger ermöglichen durch Implementierung international 
etablierter Versorgungs- und Qualitätsstandards eine Betreuung bis zum Lebensende. Altern, Pflegebedarf, Gebrechen, Sterben und Tod verschwinden durch komplette Institutionalisierung (,Verheimung") und ggf. durch die Überführung der hilfe- und pflegebedürftigen Menschen in kostengünstigere Drittländer weitgehend aus dem Bewusstsein der Bevölkerung und sind im gesellschaftlichen Alltag nicht mehr präsent.

\section{Schlussbetrachtungen}

Der vorliegende Beitrag skizziert einen Szenarioprozess zur Zukunft der Pflege. Die Teilnehmer der „Zukunftsreise“ haben sich dazu, systematisch geleitet, über die Grenzen der aktuell vorliegenden Faktenlage hinausbewegt. Das Ziel der „Zukunftsreise“ besteht weder darin, die Zukunft von gesellschaftlich relevanten Entwicklungen konkret vorherzusagen, noch darin, eine Bewertung der generierten Zukunftsszenarien herbeizuführen, um die Realisierung von wünschenswert erachteten Zustände vorzubereiten. Es geht vielmehr darum, die Bandbreite möglicher Entwicklungen aufzuzeigen und damit den Blick für zukünftige Problemstellungen zu schärfen und Anregungen für Handlungsoptionen zu liefern.

Die Vorstellung der Ergebnisse der „Reise in die Zukunft der Pflege - 2053“ wurde im vorliegenden Beitrag auf die Betrachtung der Entwicklungsphasen und der zwei konträren Szenarien zur Zukunft der Pflege beschränkt. Für die Vorstellung des Trend-Szenarios und die bildhafte Beschreibung der Szenarien, die auch Teil der Szenarien-Interpretation gemäß Phase 6 sind, wird auf weiterführende Veröffentlichungen verwiesen (Wiemann et al. 2015a und b). Weiterhin wird auf Ausführungen zu den Rückwirkungen der identifizierten Szenarien auf die gegenwärtige Situation zunächst verzichtet, da die Materialbasis zu den Phasen „Störfallanalyse“, „Konsequenzenanalyse" und ,Maßnahmenplanung" (vgl. Schritte 7 und 8 in Abb. 1) weiter zu verdichten ist, um zu belastbaren Aussagen zu gelangen. Eine jüngst angelaufene Studie an der Pflegewissenschaftlichen Fakultät der Philosophisch-Theologischen Hochschule Vallendar wird diesbezüglich substanzielle Beiträge zu gewinnen suchen, indem die Diskussionsgrundlage durch Einbindung von öf- fentlichen und fachöffentlichen Diskursen deutlich verbreitert werden soll.

$\mathrm{Zu}$ den wesentlichen Erkenntnissen des vorliegenden Beitrags wird dagegen gezählt, dass - unabhängig von der konkreten Belastbarkeit der hier generierten Szenarien - die Zukunft der Pflege in Deutschland voraussichtlich ganz erheblich mit gesamtgesellschaftlichen Umwälzungen verknüpft sein wird. Die Zukunft der Pflege ist abhängig von in sich komplexen und komplex zusammenwirkenden Faktoren, die sich in der gesellschaftlichen Entwicklung insgesamt, insbesondere aber in der wirtschaftlichen sowie der wissenschaftlich-technologischen Entwicklung begründen. In welche Richtung sich die Gesellschaft bewegen will - sei es in Richtung einer leistungs-, vitalitäts- und gesundheitsorientierten, sei es in Richtung einer zivilgesellschaftlich und sozial orientierten Gesellschaft - wird wesentlich davon abhängen, ob es gelingt, Fragen der langfristigen Entwicklung öffentlich und nachhaltig zur Diskussion zu stellen, Zukunftsgestaltung also zu einer öffentlichen Aufgabe zu machen.

Szenarien der ästhetischen und gesundheitlichen Selbstüberwachung und Selbstkorrektur im Sinne der Selbstoptimierung konkretisieren und realisieren sich derzeit bereits ebenso (vgl. Harrasser 2013) wie Entwürfe der „Sorgenden Gemeinschaft" (vgl. Klie 2014). Auch die damit verbundenen Herausforderungen werden deutlicher: Während der eine Trend Selbstoptimierung ins kollektive Bewusstsein hebt und auf diese Weise einen Kulturwandel vorantreibt, der die Freiheit des Individuums oberflächlich betrachtet zwar erweitert, letztlich aber Handlungszwänge (auch im Bereich der Pflege) entlang normativer gesellschaftlicher Kategorien deutlich verschärft, sucht der gegenläufige Entwurf Pflegearbeit in eine beziehungs- und alltagsorientierte, zivilgesellschaftlich aufzufangende Sorgearbeit und eine spezialisierte und problemlösende, professionell zu erbringende Versorgungsarbeit aufzuspalten und verliert damit das Potenzial einer Pflegearbeit aus dem Blick, für das Wohlergehen der Hilfeempfänger konstitutive Transformationsleistungen an der Schnittstelle von Gesundheitssystem und Lebenswelt zu erbringen. Die Möglichkeiten und Begrenzungen der derzeit anvisierten Zukünfte der Pflege im Lichte der mög- 
lichen Zukünfte zu reflektieren und vor diesem Hintergrund Handlungsoptionen für eine „Gesellschaft des langen Lebens" auszuloten, bleibt damit eine zentrale Aufgabe.

\section{Anmerkung}

1) Das Projekt wurde mit Mitteln des Bayerischen Finanzministeriums, der Hans Sauer Stiftung, des Vincentz Verlags und der deep innovation $\mathrm{GmbH}$ finanziert. Weitere Informationen zum Projekt „Reise in die Zukunft der Pflege - 2053“ sind über http://www.zukunftsreise-pflege.de und über die Projektpartner deep innovation $\mathrm{GmbH}$ München, Lehrstuhl für Gemeindenahe Pflege der Philosophisch-Theologischen Hochschule Vallendar, mcquadrat Berlin und v. Reibnitz St Paul - de Vence zugänglich.

\section{Literatur}

Beckert, B.; Goluchowicz, K.; Kimpeler, S. et al., 2008: Die IT- und Medienwelt in Baden-Württemberg im Jahr 2020. Vier Basisszenarien. Stuttgart

Brandenburg, H.; Güther, H.; Proft, I. (Hg.), 2015: Kosten kontra Menschlichkeit. Herausforderungen an eine gute Pflege im Alter. Ostfildern

deep innovation $\mathrm{GmbH}$ (Hg.), 2015a: Reise in die Zukunft der Pflege - 2053. Autoren: Wiemann, B.; Hülsken-Giesler, M.; Diemer, R. et al. München

deep innovation $\mathrm{GmbH}$ (Hg.), 2015b: Pfade in die $\mathrm{Zu}$ kunft der Pflege - Ausgewählte Handlungsräume in Bayern. Autoren: Wiemann, B.; Hülsken-Giesler, M.; Diemer, R. et al. München

Harrasser, K., 2013: Körper 2.0. Über die technische Erweiterbarkeit des Menschen. Bielefeld

Klie, T., 2014: Wen kümmern die Alten? Auf dem Weg in eine sorgende Gemeinschaft. München

Kosow, H.; Gaßner, R.; Erdmann, L. et al., 2008: Methoden der Zukunfts- und Szenarioanalyse. Überblick, Bewertung und Auswahlkriterien. Berlin

Manzei, A.; Schmiede, R. (Hg.), 2014: 20 Jahre Wettbewerb im Gesundheitswesen. Wiesbaden

MSW NRW - Ministerium für Schule und Weiterbildung des Landes Nordrhein-Westfalen (Hg.), o. J.: Die Szenario-Methode. Grundgedanke, Ziele, Merkmale, Typen. Landesprogramm Bildung und Gesundheit; http://www.bug-nrw.de/cms/upload/pdf/struktur. pdf (download 2.7.15)
Popp, R.; Schüll, E. (Hg.), 2009: Zukunftsforschung und Zukunftsgestaltung. Beiträge aus Wissenschaft und Praxis. Berlin

Rothgang, H.; Müller, R.; Unger, R., 2012: Themenreport „Pflege 2030“. Was ist zu erwarten - was ist zu tun? Gütersloh

Slotala, L., 2011: Ökonomisierung der ambulanten Pflege. Eine Analyse der wirtschaftlichen Bedingungen und deren Folgen für die Versorgungspraxis ambulanter Pflegedienste. Wiesbaden

$v b w$ - Vereinigung der Bayrischen Wirtschaft e.V., 2012: Pflegelandschaft 2030. Eine Studie der Prognos AG im Auftrag der vbw; http://www.prognos.com/ fileadmin/pdf/publikationsdatenbank/121000_Prognos_vbw_Pflegelandschaft_2030.pdf (download 17.2.13)

Vollmar, H.Chr. (Hg.), 2014: Leben mit Demenz im Jahr 2030. Ein interdisziplinäres Szenario-Projekt zur Zukunftsgestaltung. Weinheim

Wilms, F.E.P. (Hg.), 2006: Szenariotechnik. Vom Umgang mit der Zukunft. Bern

\section{Kontakt}

Prof. Dr. Manfred Hülsken-Giesler Pflegewissenschaftliche Fakultät Philosophisch-Theologische Hochschule Vallendar Pallottistraße 3, 56179 Vallendar E-Mail: mhuelsken-giesler@pthv.de 